COMMENT. The authors conclude that the EITB antibody-detecting assay is equally sensitive on serum and CSF samples. The ELISA assay for antigen detection is more sensitive when performed on CSF samples than serum, but less sensitive than the EITB assay. ELISA, using either serum or CSF samples, is better than EITB in the differentiation of active and inactive NCC. High antigen levels detected by ELISA suggest the presence of subarachnoid NCC, associated with a worse prognosis.

Since neuroimaging is often nonspecific for $\mathrm{NCC}$, immunodiagnosis is usually necessary for confirmation. CSF samples offer no advantage over serum for detection of antibodies by EITB assay. In intraparenchymal cases, although the use of CSF samples for antigen detection by ELISA assay may yield a $13 \%$ increase in case identification, the increase over serum samples is not significant. With extraparenchymal disease, most patients are strongly seropositive by EITB assay on either serum or CSF.

The findings in the above study suggest that serum antibody detection by an EITB assay, using purified antigen, is the assay of choice for diagnosis of NCC.

\title{
ANTI-N-METHYL-D-ASPARTATE RECEPTOR ENCEPHALITIS WITH CATATONIA TREATED BY PLASMAPHERESIS
}

The case of a 12-year-old girl with anti-methyl-D-aspartate receptor (NMDAR) encephalitis is reported by researchers from Augsburg and Bonn, Germany, and Oxford, UK. She was admitted with an episode of paresthesia and hypotonia of the left leg, and rare episodes of head turning with clonic movements of the left and all extremities. The symptoms were preceded by a 2-day episode of diarrhea 3 weeks before. The EEG, brain MRI, and CSF were normal, and she was discharged with a suspected diagnosis of psychogenic seizures. She was readmitted 2 days later with agitation, hyperventilation and intermittent ocular deviation, followed by intermittent catatonic postures with fever, hyperidrosis, chewing movements and tongue protrusion. She required tube feeding and intermittent oxygen. EEG now showed continuous slowing. The MRI was unremarkable. IgM antibodies against Campylobacter jejuni were elevated. Prednisolone was ineffective. At 6 weeks after admission, CSF showed IgG antibody reactivity with hippocampal neurophil, and subsequently, serum antibodies to NMDAR were demonstrated. Plasmapheresis was followed within 2 weeks by speaking words, walking and almost full recovery in 4 weeks. CSF antibody reactivity was no longer detected, and ultrasound and CT were negative for teratoma in the pelvis and mediastinum. (Schimmel M, Bien CG, Vincent A, Schenk W, Penzien J. Successful treatment of anti-N-methyl-D-aspartate receptor encephalitis presenting with catatonia. Arch Dis Child April 2009;94:314-316). (Respond: Dr Johannes Penzien, Department of Paediatrics, Klinikum Augsburg, Stenglinstrasse 2, 86156 Augsburg, Germany. E-mail: johann.penzien@klinikum.augsburg.de).

COMMENT. This 12-year-old girl appears to be the youngest patient reported with anti-NMDAR encephalitis. Her presentation with neuropsychiatric symptoms progressing to seizures, catatonia, autonomic dysfunction, hypoventilation, orofacial dyskinesia and dysphagia is typical. Teratoma, absent in this child, may occur in $65 \%$ of patients, and sometimes develops 7 years following encephalitis (Dalmau $\mathrm{J}$ et al. Lancet Neurol 2008;7:327-340)(Iizuka T et al. Neurology 2008;70:504-511). An apparent response to plasmapheresis and the association with Campylobacter infection are of interest. 